\title{
HOLOMORPHIC MAPPINGS INTO STRICTLY CONVEX DOMAINS WHICH ARE KOBAYASHI ISOMETRIES AT ONE POINT
}

\author{
IAN GRAHAM \\ (Communicated by Irwin Kra)
}

\begin{abstract}
Let $M$ be a complex manifold of dimension $n$ and let $\Omega$ be a domain in $\mathbf{C}^{n}$. Let $f: M \rightarrow \Omega$ be a holomorphic map which is an isometry for the infinitesimal Kobayashi metric at one point. We given conditions on $M$ and on $\Omega$ which imply that $F$ must be a biholomorphic map.
\end{abstract}

\section{INTRODUCTION AND STATEMENT OF RESULTS}

The purpose of this paper is to prove the following theorem:

Theorem. Suppose $M$ is a taut complex manifold of dimension $n$. Suppose $\Omega$ is a strictly convex bounded domain in $\mathbf{C}^{n}$. Suppose $F: M \rightarrow \Omega$ is a holomorphic mapping which is a Kobayashi isometry at a point $p \in M$. Then $F$ is biholomorphic.

This theorem is in a sense dual to the following result of Vigue [12, Theorem 3.1]:

Theorem (Vigué). Suppose $\Omega$ is a bounded convex domain in $\mathbf{C}^{n}$ and suppose $M$ is a complex manifold on which the Carathéodory pseudodistance is a distance. Suppose $F: \Omega \rightarrow M$ is a holomorphic mapping which is a Carathéodory isometry at a point $p \in \Omega$. Then $F$ is biholomorphic.

Vigue himself proved a result along the lines of ours [12, Theorem 4.3], but with a much stronger assumption on the domain of the mapping (the set of holomorphic maps from the unit disc to $M$ which realize the Kobayashi length of some vector $\xi \in T_{p} M$ should fill up $M$ ). However, his assumptions on the range of the mapping are weaker-for example, convexity rather than strict convexity is sufficient.

Our proof makes use of the properties of extremal maps for the Kobayashi metric on strictly convex domains established by Lempert [5].

Received by the editors June 2, 1988.

1980 Mathematics Subject Classification (1985 Revision). Primary 32H20.

The author was partially supported by a grant from the Natural Sciences and Engineering Research Council of Canada. 


\section{Definitions and basic Facts}

In this section we recall some well-known definitions:

Let $M$ be a connected complex manifold. Let $D$ be the unit disc in $\mathbf{C}$. Let $p \in M$, and let $\xi \in T_{p} M$. The Kobayashi length of $\xi$ is

$$
\begin{aligned}
& K(p ; \xi)=\inf \left\{|v| \mid v \in T_{0} D\right. \text { and there exists a holomorphic map } \\
& \left.\qquad: D \rightarrow M \text { such that } \phi(0)=p \text { and } d \phi_{0}(v)=\xi\right\} .
\end{aligned}
$$

By the well-known work of Royden [8], if the Kobayashi length of a parametrized piecewise-smooth curve $\gamma:[a, b] \rightarrow M$ is defined by

$$
l_{K}(\gamma)=\int_{a}^{b} K\left(\gamma(t) ; \gamma^{\prime}(t)\right) d t
$$

then the formula

$$
K(p, q)=\inf \left\{l_{K}(\gamma) \mid \gamma \text { joins } p \text { to } q\right\}
$$

gives the Kobayashi distance, or rather pseudo-distance, between $p$ and $q$. (Kobayashi originally gave a different construction [3].)

$M$ is called hyperbolic if $K(p, q)>0$ whenever $p \neq q$, and complete hyperbolic if $K($,$) is a complete metric. M$ is called taut [14] if whenever $N$ is a complex manifold and $\phi_{j}: N \rightarrow M$ is a sequence of holomorphic mappings, then either there exists a subsequence which is compactly divergent or a subsequence which converges uniformly on compact subsets to a holomorphic map $\phi: N \rightarrow M$. It sufficies that this condition should hold when $N=D$ in order for $M$ to be taut [1]. Also a complete hyperbolic manifold is taut, and a taut manifold is hyperbolic. If $M$ is taut the infimum in (2.1) is always assumed for some mapping $\phi$. Any such $\phi$ is said to be extremal for $K(p ; \xi)$.

With $\xi \in T_{p} M$ as before, the Carathéodory length of $\xi$ is

$$
C(p ; \xi)=\sup \left\{\left|d g_{p}(\xi)\right| \mid g: M \rightarrow D \text { holomorphic, } g(p)=0\right\} \text {. }
$$

Similarly we define

$$
l_{C}(\gamma)=\int_{a}^{b} C\left(\gamma(t) ; \gamma^{\prime}(t)\right) d t
$$

and

$$
\tilde{C}(p, q)=\inf \left\{l_{C}(\gamma) \mid \gamma \text { joins } p \text { to } q\right\} .
$$

The Carathéodory pseudo-distance is defined by

$$
C(p, q)=\sup \{\rho(g(p), g(q)) \mid g: M \rightarrow D \text { holomorphic }\}
$$

where $\rho$ is the Poincare distance. In general, $C(p, q) \leq \widetilde{C}(p, q)$, and strict inequality is possible.

When appropriate we shall indicate the manifold on which we are considering these metrics by a subscript, e.g., $K_{M}(p ; \xi)$. A holomorphic map $F: M \rightarrow N$ is a Kobayashi isometry at a point $p \in M$ if $K_{M}(p ; \xi)=K_{N}\left(F(p) ; d F_{p}(\xi)\right)$ for all $\xi \in T_{p} M$. Similarly $F$ is a Carathéodory isometry at $p$ if $C_{M}(p ; \xi)=$ $C_{N}\left(F(p) ; d F_{p}(\xi)\right)$ for all $\xi \in T_{p} M$. 
A holomorphic map $\phi: D \rightarrow M$ is called a complex geodesic for the Carathéodory metric if

$$
C_{M}\left(\phi\left(z_{1}\right), \phi\left(z_{2}\right)\right)=C_{D}\left(z_{1}, z_{2}\right)=\rho\left(z_{1}, z_{2}\right)
$$

for all $z_{1}, z_{2} \in D$, i.e., $\phi$ is an isometry (globally) for the Carathéodory distance.

This definition is due to Vesentini [10]. It is worth remarking on its relation to the concept of "metrische Ebene" or metric plane as used by Carathéodory and Reiffen. Carathéodory [2, p. 103], working primarily on the bidisc, defined a metrische Ebene to be a one-dimensional submanifold $A$ of $M$ such that $C_{A}(p, q)=C_{M}(p, q)$ for all $p, q \in A$. Reiffen [7, p. 19], working on general complex manifolds, explicitly added the requirement that $A$ should be biholomorphic to $D$ and characterized such objects as holomorphic retracts of $M$. Thus a metrische Ebene is precisely the range of a complex geodesic.

Of course, complex geodesics need not exist for a given complex manifold, but if they do exist they have strong properties [10]. In particular, Vesentini observed that if a holomorphic map $\phi: D \rightarrow M$ is a Carathéodory isometry at one point $z_{0}$, then $\phi$ must be complex geodesic. This implies the following fact, which was exploited by Vigue [12] in proving the theorem quoted above: if $F: M \rightarrow N$ is a holomorphic mapping between complex manifolds which is a Carathéodory isometry at a point $p \in M$, and if $\phi: D \rightarrow M$ is a complex geodesic passing through $p$, then $F \circ \phi$ is a complex geodesic passing through $F(p)$.

Similarly, a complex geodesic for the Kobayashi metric is a holomorphic map $\phi: D \rightarrow M$ which is an isometry (globally) for the Kobayashi distance [10]. A holomorphic map $\phi: D \rightarrow M$ which is extremal for some $K(p ; \xi)$ need not be a complex geodesic for the Kobayashi metric, but it is if $M$ is a convex bounded domain in $\mathbf{C}^{n}[5,9]$. In this case, when $\partial M$ is smooth the boundary values of extremal maps were characterized by Lempert [5] (see also Royden and Wong [9]); with this additional characterization extremal maps for the Kobayashi metric are called stationary maps in this context.

A complex geodesic for the Caratheodory metric is a complex geodesic for the Kobayashi metric, and $C()=,\widetilde{C}()=,K($,$) along its image [10].$

Finally, we recall that a domain $\Omega \subset \mathbf{C}^{n}$ is called strictly convex if whenever $L$ is a closed line segment such that $L \subset \bar{\Omega}$ we have $L^{\circ} \subset \Omega$.

\section{EXtremal MAPS FOR THE Kobayashi Metric ON $M$}

We first suppose that $M$ is taut and that $\Omega$ is a bounded convex domain in $\mathrm{C}^{n}$ (rather than strictly convex). We suppose that $F: M \rightarrow \Omega$ is a holomorphic map which is a Kobayashi isometry at $p \in M$. Under these conditions we have

Proposition 1. (a) Let $\xi \in T_{p} M$ and suppose that $\phi: D \rightarrow M$ is an extremal map for $K(p ; \xi)$. Then $\phi$ is a complex geodesic for the Kobayashi metric, and hence $\phi$ is a proper map and $\phi(D)$ is an embedded disc. 
(b) If, furthermore, $\Omega$ is strictly convex, and if $\phi_{1}$ and $\phi_{2}$ are extremal maps for $K_{M}\left(p ; \xi_{1}\right)$ and $K_{M}\left(p ; \xi_{2}\right)$, then either $\phi_{1}(D) \cap \phi_{2}(D)=\{p\}$ or else there exists $\theta \in \mathbf{R}$ such that $\phi_{1}\left(e^{i \theta} z\right)=\phi_{2}(z)$ for all $z \in D$.

Proof. (a) Since $F$ is a Kobayashi isometry at $p, F \circ \phi$ is extremal for $K_{\Omega}\left(F(p) ; d F_{p}(\xi)\right)$. Hence by the work of Lempert [5] and Royden and Wong [9], $F \circ \phi$ is a complex geodesic. The distance-decreasing property shows that $\phi$ itself must be a complex geodesic. If $\left\{z_{j}\right\} \subset D$ is a sequence such that $\left|z_{j}\right| \rightarrow 1$, then $K\left(p, \phi\left(z_{j}\right)\right)=\rho\left(0, z_{j}\right) \rightarrow \infty$. This shows that $\phi$ is proper. The face that $\phi$ is an isometry similarly implies that it is $1-1$ with nonvanishing differential.

(b) If $\phi_{1}$ and $\phi_{2}$ are extremal maps for $K_{M}\left(p ; \xi_{1}\right)$ and $K_{M}\left(p ; \xi_{2}\right)$, then $F \circ \phi_{1}$ and $F \circ \phi_{2}$ are extremal maps for $K_{\Omega}\left(F(p) ; d F_{p}\left(\xi_{1}\right)\right)$ and $K_{\Omega}(F(p)$; $\left.d F_{p}\left(\xi_{2}\right)\right)$. Hence either $F \circ \phi_{1}(D) \cap F \circ \phi_{2}(D)=\{F(p)\}$ or else there exists $\theta \in \mathbf{R}$ such that $F \circ \phi_{1}\left(e^{i \theta} z\right)=F \circ \phi_{2}(z)$ for all $z \in D$ [5]. In the first case we must have $\phi_{1}(D) \cap \phi_{2}(D)=\{p\}$ since $\phi_{j}$ and $F \circ \phi_{j}$ are isometries, $j=1,2$. In the second case we have $\phi_{1}\left(e^{i \theta} z\right)=\phi_{2}(z)$ in a neighborhood of 0 (since $F$ is biholomorphic in a neighborhood of $p$ ), hence everywhere in $D$ by analytic continuation.

\section{A PROPERTY OF COMPLEX GEODESICS}

Proposition 2. Let $M$ be a taut complex manifold. Let $S$ (resp., $T$ ) be the set of points which can be joined to a point $p \in M$ by a complex geodesic for the Kobayashi metric (resp., by a complex geodesic for the Carathéodory metric). Then $S$ (resp. $T$ ) is closed in $M$.

Proof. Suppose $\left\{p_{j}\right\}_{j=1}^{\infty} \subset S, p_{j} \rightarrow \tilde{p} \in M$. Let $\phi_{j}: D \rightarrow M$ be complex geodesics and $r_{j} \in D, 0 \leq r_{j}<1$ be points such that $\phi_{j}(0)=p, \phi_{j}\left(r_{j}\right)=p_{j}$, $j=1,2, \ldots$. Since $M$ is taut and $\phi_{j}(0)=p$ for all $j$ there is a subsequence, which we again denote by $\phi_{j}$, and a holomorphic map $\phi: D \rightarrow M$ such that $\phi_{j} \rightarrow \phi$ uniformly on compact subsets. Since $K(p, \tilde{p})<\infty$ it follows that $\left\{K\left(p, p_{j}\right)\right\}$ are bounded, hence that $\left\{r_{j}\right\}_{j=1}^{\infty}$ lies in a compact subset of $D$. A subsequence of the $\left\{r_{j}\right\}$, which we again denote by $\left\{r_{j}\right\}$, tends to $r<1$. It is easy to see that $\phi$ is a complex geodesic and that $\phi(r)=\tilde{p}$. Mutatis mutandis we obtain the same result for $T$.

\section{Proof of The TheOREM}

Let $M_{0}=\cup\{\phi(D) \mid \phi: D \rightarrow M$ is a complex geodesic for the Kobayashi metric and $\phi(0)=p\}$. Let $f=F_{\mid M_{0}}$. A priori $f$ need not be holomorphic, since $M_{0}$ is so far only a subset of $M$. However, using Proposition 1 and the fact that the extremal discs of $\Omega$ fill up $\Omega$ [5], we see that $f$ gives a 1-1 map of $M_{0}$ onto $\Omega$. By Proposition $2, M_{0}$ is closed in $M$. If we can show that it is open, that it must coincide with $M$, which would show that $F$ is $1-1$ and finish the proof.

To show that $M_{0}$ is open it suffices to show that $f$ is a homeomorphism (with the induced topology on $M_{0}$ ). The continuity of $f$ is immediate, so we need 
only show that $f^{-1}$ is continuous. Let $\left\{q_{j}\right\}_{j=1}^{\infty} \subset \Omega$ and suppose $\left\{q_{j}\right\} \rightarrow \tilde{q} \in \Omega$. Let $p_{j} \in M_{0}$ be the unique point such that $f\left(p_{j}\right)=q_{j}, j=1,2, \ldots$. Let $\phi_{j}: D \rightarrow M$ be complex geodesics for the Kobayashi metric and $r_{j} \in D, 0 \leq$ $r_{j}<1$ be points such that $\phi_{j}(0)=p, \phi_{j}\left(r_{j}\right)=p_{j}, j=1,2, \ldots$ By the tautness of $M$ there exists a subsequence $\phi_{j_{k}}$ and a holomorphic map $\phi: D \rightarrow M$ such that $\phi_{j_{k}} \rightarrow \phi$ uniformly on compact subsets. The distances $\rho\left(0, r_{j_{k}}\right)=$ $K_{M}\left(p_{j_{k}}, p\right) \stackrel{K_{\Omega}}{=}\left(q_{j_{k}}, f(p)\right)$ are bounded, so a subsequence $r_{j_{k_{l}}}$ converges to $\tilde{r}<1$. As in Proposition 2, $\tilde{\phi}$ is a complex geodesic and $p_{j_{k_{l}}} \rightarrow \phi(\tilde{r})=\tilde{p}$. Thus $\tilde{p} \in M_{0}$, and furthermore $f(\tilde{p})=\tilde{q}$ by the continuity of $f$. The properties $\tilde{p} \in$ $M_{0}$ and $f(\tilde{p})=\tilde{q}$ determine $\tilde{p}$ uniquely, hence independently of the choices of of subsequences. It must therefore be true that $p_{j} \rightarrow \tilde{p}$, demonstrating that $f^{-1}$ is continuous and completing the proof.

\section{ACKNOWLEDGMENTS}

This paper was written during the author's sabbatical year at Standord University. The author wishes to thank the Stanford Mathematics Department for its warm hospitality, and $\mathrm{H}$. Wu for several discussions.

\section{REFERENCES}

1. T. Barth, Taut and tight complex manifolds, Proc. Amer. Math. Soc. 24 (1970), 429-431.

2. C. Carathéodory, Über eine spezielle Metrik, die in der Theorie der analytischen Funktionen auftritt, Atti Pontif. Acad. Sci. Nuovi Lincei 80 (1927), 99-105.

3. S. Kobayashi, Hyperbolic manifolds and holomorphic mappings, Marcel Dekker, New York, 1970.

4. __ Intrinsic distances, measures, and geometric function theory, Bull. Amer. Math. Soc. 82 (1976), 357-416.

5. L. Lempert, La métrique de Kobayashi et la representation des domaines sur la boule, Bull. Soc. Math. France 109 (1981), 427-474.

6. Holomorphic retracts and intrinsic metrics in convex domains, Anal. Math. 8 (1982), 257-261.

7. H. J. Reiffen, Die differentialgeometrischen Eigenschaften der invarianten Distanzfunktion von Carathéodory, Schriftenreihe Math. Inst. Univ. Münster, no. 26 (1963).

8. H. L. Royden, Remarks on the Kobayashi metric, Several Complex Variables II (Proc. Intern. Conf. Univ. of Maryland, 1970), Lecture Notes in Mathematics, vol. 185, Springer-Verlag, New York, Heidelberg and Berlin 1971, pp. 125-137.

9. H. L. Royden and P. M. Wong, Carathéodory and Kobayashi metrics on convex domains, preprint.

10. E. Vesentini, Complex geodesics, Compositio Math. 44 (1981), 375-394.

11. __ Complex geodesics and holomorphic maps, Sympos. Math. 26 (1982), 211-230.

12. J. P. Vigué, Caractérisation des automorphismes analytiques d'un domaine convexe borné, C. R. Acad. Sci. Paris 299 (1984), 101-104.

13. _. Sur la caractérisation des automorphismes analytiques d'un domaine borné, Portugaliae Math. 43 (1985-1986), 439-453.

14. H. Wu, Normal families of holomorphic mappings, Acta Math. 119 (1967), 194-233.

Department of Mathematics, Stanford University, Stanford, California 94305

Current address: Department of Mathematics, University of Toronto, Ontario, Canada M5S 1Al 